\title{
Polymer photonic crystal fibre for sensor applications
}

\author{
David J. Webb \\ Photonics Research Group, Aston University, Birmingham, B4 7ET
}

\begin{abstract}
Polymer photonic crystal fibres combine two relatively recent developments in fibre technology. On the one hand, polymer optical fibre has very different physical and chemical properties to silica. In particular, polymer fibre has a much smaller Young's modulus than silica, can survive higher strains, is amenable to organic chemical processing and, depending on the constituent polymer, may absorb water. All of these features can be utilised to extend the range of applications of optical fibre sensors. On the other hand, the photonic crystal - or microstructured - geometry also offers advantages: flexibility in the fibre design including control of the dispersion properties of core and cladding modes, the possibility of introducing minute quantities of analyte directly into the electric field of the guided light and enhanced pressure sensitivity. When brought together these two technologies provide interesting possibilities for fibre sensors, particularly when combined with fibre Bragg or long period gratings. This paper discusses the features of polymer photonic crystal fibre relevant to sensing and provides examples of the applications demonstrated to date.
\end{abstract}

Keywords: photonic crystal fibre, sensor, polymer fibre, mPOF

\section{INTRODUCTION}

The combination of polymeric optical fibre (POF) with photonic crystal waveguiding brings together two fibre technologies that can extend the sphere of application of fibre sensors. Discussion of this technology cannot be made without reference to the pioneering work at Sydney University (formerly the Optical Fibre Technology Centre). This Australian group was the first to develop single mode photonic crystal polymer fibre as well as fibres that were multicore, highly birefringent, graded in index or hollow core ${ }^{1}$. As will be described later, they were also the first to begin developing applications for this technology. They refer to photonic crystal POF as microstructured POF, or mPOF, and this name has become common place in the polymer fibre community and consequently we will use it here. In this paper we describe the properties of photonic crystal fibres and POF and see how these have been brought together to extend the range of applications of optical fibre sensors.

\subsection{Photonic Crystal Fibre}

Photonic crystal fibres (PCFs), first proposed and demonstrated in the $1990 \mathrm{~s}^{2}$, are a class of fibre in which light guiding is facilitated by an array of holes running along the length of the fibre. Two fundamentally different modes of light guidance have been identified. With index guiding fibre, the core of the fibre is a region where one or more holes are missing from an otherwise periodic two dimensional array. The core region has a refractive index almost equal to that of the fibre material, whereas the surrounding cladding region has an average index somewhere between that of the fibre material and that of the holes. This arrangement behaves rather like a conventional step index fibre in which a high index core is surrounded by a lower index cladding. There are some important differences though, for example index guiding PCF can be single mode at all wavelengths ${ }^{3}$.

The second type of PCF is photonic bandgap fibre ${ }^{4}$. In this type of fibre, light in a certain wavelength range is confined to the core due to the presence of a photonic bandgap provided by the structure of the cladding region. An important feature of photonic bandgap fibre is that with this guidance mechanism, it is not necessary to have the refractive index of the core region higher than that of the cladding region and indeed it is possible to confine light predominantly within a completely hollow core. This configuration provides considerable overlap of the guided light with any gas or liquid contained within the holes with such an arrangement permitting a long interaction length for a small total volume of analyte.

Optical Sensing and Detection, edited by Francis Berghmans, Anna Grazia Mignani, Chris A. van Hoof, Proc. of SPIE Vol. 7726, 77260Q - (C) 2010 SPIE · CCC code: 0277-786X/10/\$18 - doi: 10.1117/12.859090 


\subsection{Polymer optical fibre}

Polymer optical fibres have a well established use as a low cost alternative to silica fibre in short range telecommunications systems, e.g. local area networks, where the much higher attenuation of polymers is not so problematic. The cost advantage mainly comes from the ease of installation of the POF fibre, which is simple to cleave and connectorise and is highly flexible, even with the standard $1 \mathrm{~mm}$ diameter ${ }^{5}$. The use of POF for sensing has also been explored $^{6}$; often low cost applications are targeted, which utilise intensity based sensing approaches.

Another motivation for the use of POF is to take advantage of its very different material properties as compared to silica fibre. The Young's modulus of POF is typically some 25 times smaller than that of silica ${ }^{7-8}$. This means that it takes 25 times less force to produce a given strain in POF than it does in silica. This property is important when strain sensing on or in structures that themselves are elastic. In such circumstances the stiff silica fibre can act to reinforce the material, reporting a much lower strain than would occur without the presence of the fibre.

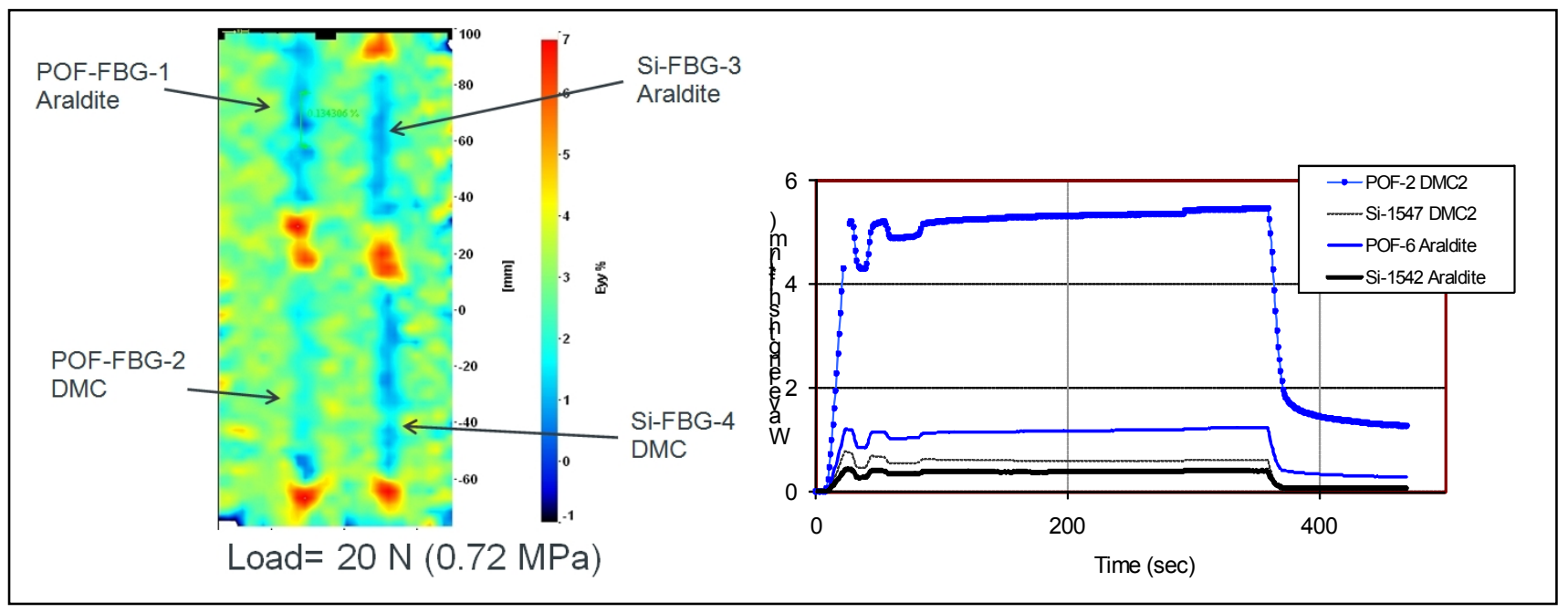

Figure 1 Left: Strain field of woven cloth imaged by digital image correlation. Right: response of FBGs on cloth to application of load.

Figure 1 illustrates an example of this behaviour. As part of a project investigating optical engineering approaches to monitoring historic tapestries as an aid to their conservation, fibre Bragg gratings (FBGs - described in more detail later on) in silica fibre and POF were fixed to a woven textile, which was then placed under a $20 \mathrm{~N}^{\circ} \mathrm{oad}^{9}$. The strain in the direction of the load was determined over the structure using digital image correlation. It may be seen in the resulting image shown on the left of Figure 1 that the POF FBG fixed using the elastic conservation adhesive DMC perturbs the strain field much less than either the silica FBGs or the other POF FBG fixed using the much stiffer Araldite adhesive. This behaviour is made clear in the right hand plot which shows the wavelength shifts recovered from the FBGs as the cloth was loaded and unloaded. As will be quantified later on, the sensitivity of silica and POF FBGs to strain are fairly close and consequently the figure implies the POF FBG fixed with DMC adhesive is actually experiencing much greater strain that the other sensors.

The second important feature of POF is its ability to survive high strains ${ }^{10}$. The visco-elastic nature of POF can lead to problems at higher strains ${ }^{11}$, nevertheless hysteresis free behaviour at strains of over $10 \%$ have been reported ${ }^{12}$. There are other properties of POF that can also be used to advantage in sensor systems. Polymers are organic materials typically drawn to fibre at a temperature of around $200{ }^{\circ} \mathrm{C}$, which is low enough not to denature organic materials. As a consequence, it is possible to modify the fibre material prior to drawing, either directly using organic chemical techniques, or by inserting an organic dopant. Finally, some polymers, e.g. PMMA, possess an affinity for water, which changes their optical properties on absorption ${ }^{13}$. 


\section{EXAMPLE APPLICATIONS}

\subsection{Sensing within the fibre holes}

The holes in MPOF provide the opportunity to promote light interaction with small volumes of liquid, gas or chemical or biochemical species over significant lengths. Before providing some example applications of this approach it is worth noting that at the preform stage, the holes also offer a convenient means of introducing dopants into the polymer. In an mPOF preform produced by drilling holes into a cylinder of PMMA, the hole diameter will be typically of the order of 1 $\mathrm{mm}$. In the first demonstration of solution doping a two stage drawing process was used with the liquid being added after the first stage following annealing of the resulting preform when the holes were approximately $250 \mu \mathrm{m}$ in diameter ${ }^{14}$. At this scale it is still very easy to fill the holes selectively with liquid, which in this case was Rhodamine 6G dye dissolved in methanol. Diffusion is relatively slow with three days being required to fill the core of the fibre with dopant. Successful doping was confirmed by fluorescence measurements and the process was later used to create a dye laser.

A similar approach has been used to realise a $\mathrm{pH}$ sensor ${ }^{15}$. In this case, a relatively large fibre was fabricated of diameter $760 \mu \mathrm{m}$ containing two rings of holes with diameter $100 \mu \mathrm{m}$. The holes were filled with a solution of eosin and cellulose acetate dissolved in acetic acid, which after drying formed an eosin doped cellulose acetate film on the hole surface. The fluorescence from the film was used to monitor the $\mathrm{pH}$ of solutions introduced into the holes. A photonic bandgap $\mathrm{mPOF}$ was used in an experiment that demonstrated surface enhanced Raman spectroscopy inside the holes of a kagome lattice style fibre ${ }^{16}$. An important advantage of the photonic bandgap fibre in this application is that waveguiding is still possible when the holes are filled with liquid. For this demonstration Rhodamine $6 \mathrm{G}$ was adsorbed onto colloidal silver particles in the core of the fibre. The same group have also recently demonstrated the first surface plasmon resonance sensor using $\mathrm{mPOF}^{17}$.

The first biosensing experiments using $\mathrm{mPOF}$ were carried out using a simple, highly multimode PMMA structure consisting of just six $60 \mu \mathrm{m}$ holes surrounding the fibre $\operatorname{core}^{18}$. The holes were filled with a phosphate buffered saline solution of streptavidin, which bound to the PMMA. The authors were able to demonstrate that the sensor responded selectively to $\alpha$-streptavidin. The binding of the $\alpha$-streptavidin was detected by attaching Cy 3 fluorophores to the $\alpha$ streptavidin and illuminating the fibre from the side using $532 \mathrm{~nm}$ laser light - fluorescence was guided by the fibre and detected via a multimode silica down-lead to a spectrometer. The authors have further developed this work making use of photonic crystal fibres fabricated from TOPAS cyclic olefin copolymer, rather than PMMA ${ }^{19}$. TOPAS has the useful property that it is intrinsically inert and will not readily bind to biomolecules like PMMA. The surface of TOPAS can be activated, however, by attaching Antraquinon to the surface using UV light. The exposed regions can then accept biomolecules in a similar fashion to PMMA. This process allows for the definition of specific localized sensing regions within the fibre.

\subsection{Fibre gratings}

The exploitation of the innate photosensitivity of silica fibre to realise fibre Bragg and long period gratings has revolutionised the field of optical fibre sensing over the last 20 years and FBG sensors in particular have now matured to the point where there has been commercial success in several industrial sectors, notably oil and gas and wind energy. The majority of POFs are based on poly(methyl methacrylate) and it has been known since the early nineteen seventies that this material was also sensitive to UV light ${ }^{20}$. Indeed it was around this time that the first Bragg gratings in doped PMMA were used to create a laser, albeit in bulk material rather than fibre ${ }^{21}$.

The first POF FBGs were reported about 10 years ago in step index fibre ${ }^{22}$ and a few years later in $\mathrm{mPOF}^{23}$. Photoinscribed long period gratings were first produced in step index fibre using a mecury discharge lamp ${ }^{24}$ and in mPOF using a HeCd laser ${ }^{25}$. Because of the typical scale of the index modulation needed for LPGs -100 s of microns as opposed to about half a micron for FBGs - they can be produced using alternative methods. In the case of silica fibre, LPGs can be written point-by-point using a $\mathrm{CO}_{2}$ laser $^{26}$ or an electric $\operatorname{arc}^{27}$ or simply produced by periodically bending the fibre ${ }^{28}$. This latter approach was used to produce the first LPGs in $\mathrm{mPOF}^{29}$.

Table 1 compares the sensitivities of silica and POF FBGs. It may be seen that whilst the strain sensitivities are broadly similar the temperature sensitivity of POF is about 4 times greater and of the opposite sign; the latter being due to the negative thermooptic coefficient of PMMA ${ }^{30}$. Note that early papers studying the thermal response of POF FBGs report a variety of sensitivities, often more than $100 \mathrm{pm} /{ }^{\circ} \mathrm{C}^{31}$. This is likely to be a result of confounding the influences of temperature and humidity by carrying out temperature tests under ambient laboratory conditions.

Proc. of SPIE Vol. 7726 77260Q-3 
Table 1 Sensitivities to various measurands of FBGs in silica fibre and PMMA based POF at 1550nm.

\begin{tabular}{|l|l|l|}
\hline Measurand & Silica fibre & POF (PMMA based) \\
\hline Strain & $1.2 \mathrm{pm} / \mu \varepsilon^{32}$ & $1.5 \mathrm{pm} / \mu \varepsilon^{33}$ \\
\hline Temperature & $10 \mathrm{pm} /{ }^{\circ} \mathrm{C}^{32}$ & $-43 \mathrm{pm} /{ }^{\circ} \mathrm{C}^{34}$ \\
\hline Humidity & - & $38 \mathrm{pm} / \%^{34}$ \\
\hline
\end{tabular}

With the glass transition temperature of PMMA only around $104{ }^{\circ} \mathrm{C}^{35}$, the useable temperature range of POF gratings is necessarily limited. Furthermore, even below $100^{\circ} \mathrm{C}$ care must be taken to anneal the fibre to obtain repeatable behaviour. Figure 2 illustrates this procedure. In this experiment, mPOF containing an FBG was heated 3 times, returning to room temperature after each cycle. The first cycle took the temperature to $77^{\circ} \mathrm{C}$, the second to $86^{\circ} \mathrm{C}$, and the third to $92^{\circ} \mathrm{C}$.

The results show a significant shift in the wavelength of the grating at room temperature once the grating is taken above the region of near linear response. This quasi-linear region extends to higher temperatures with each cycle (approximately equal to the maximum temperature of the previous heating cycle). Though in the figure a straight line has been fitted to this region, showing the approximate temperature sensitivities of each cycle, the response in the low temperature region for each cycle is in fact nonlinear with the greatest sensitivity at low temperatures. The deviation from the linear response is shown in the inset of Figure 2 and is likely due to the humidity sensitivity of the POF.



Figure 2 Thermal annealing behaviour of FBG in polymer $\mathrm{PCF}^{36}$. See text for details.

In the case of the first heating cycle, the wavelength begins to rapidly decrease with temperature between 60 and $65^{\circ} \mathrm{C}$. The hysteresis observed in the Bragg wavelength at $23^{\circ} \mathrm{C}$ between the first and second cycles is $8.4 \mathrm{~nm}$. The wavelength of the grating in the second heating cycle does not begin to rapidly decrease with temperature until $76^{\circ} \mathrm{C}$, close to the maximum temperature of the first cycle. The hysteresis observed at $23^{\circ} \mathrm{C}$ between the second and third cycles is $10.1 \mathrm{~nm}$. The wavelength response of the grating with temperature in the 3rd cycle followed the same trend displayed in the second cycle, with the response remaining linear, with a sensitivity of $-77 \mathrm{pm} /{ }^{\circ} \mathrm{C}$, until the maximum temperature of the second cycle was reached.

The permanent wavelength shift observed at room temperature, and the rapid decrease in wavelength with temperature after a certain temperature during heating, was explained as being due to fibre shrinkage ${ }^{37}$. Polymer optical fibre is pulled under tension, resulting in an axial orientation of the polymer chains and residual strain in the fibre. However, at 
a certain temperature, the chains are thought to relax from the axial orientation causing shrinkage of the fibre. This effect has been reported previously to be a function of the drawing tension ${ }^{38}$.

Clearly, to obtain the maximum working range it is necessary to anneal the fibre and the annealing behaviour must be taken into account in arranging for the final device to have a particular nominal Bragg wavelength. The authors of reference ${ }^{36}$ showed that it was possible to anneal the fibre prior to grating inscription for 7 hours at $80{ }^{\circ} \mathrm{C}$ and thereby obtain a hysteresis free working range of up to $90{ }^{\circ} \mathrm{C}$. This approach avoids having to cope with the permanent Bragg wavelength shift of around $20 \mathrm{~nm}$ that occurs with post-inscription annealing.



Figure 3 a) Humidity sensitivity of FBG in PMMA based POF. b) Response time of FBG to 5\% change in humidity with fibre of diameter $160 \mu \mathrm{m}$.

Unlike silica fibre, PMMA based POF is sensitive to humidity as shown in Figure 3. This high sensitivity has been exploited to detect water in aviation fuel where resolution better than $10 \mathrm{ppm}$ appears to be feasible ${ }^{39}$. The diffusion of water into the fibre can be monitored very clearly using a long period grating. Figure 4 shows the wavelength shift of one of the resonant peaks of a $2 \mathrm{~cm}$ long LPG with period $1 \mathrm{~mm}$ fabricated in single mode mPOF when the fibre is submerged in water ${ }^{25}$ (the initial instantaneous shift on submersion due to the change in refractive index surrounding the fibre is not shown). The initial red shift occurs as water diffuses into the cladding. Once the water begins to reach the core the wavelength shift reverses and by the time steady state is reached a net blue shift has occurred.

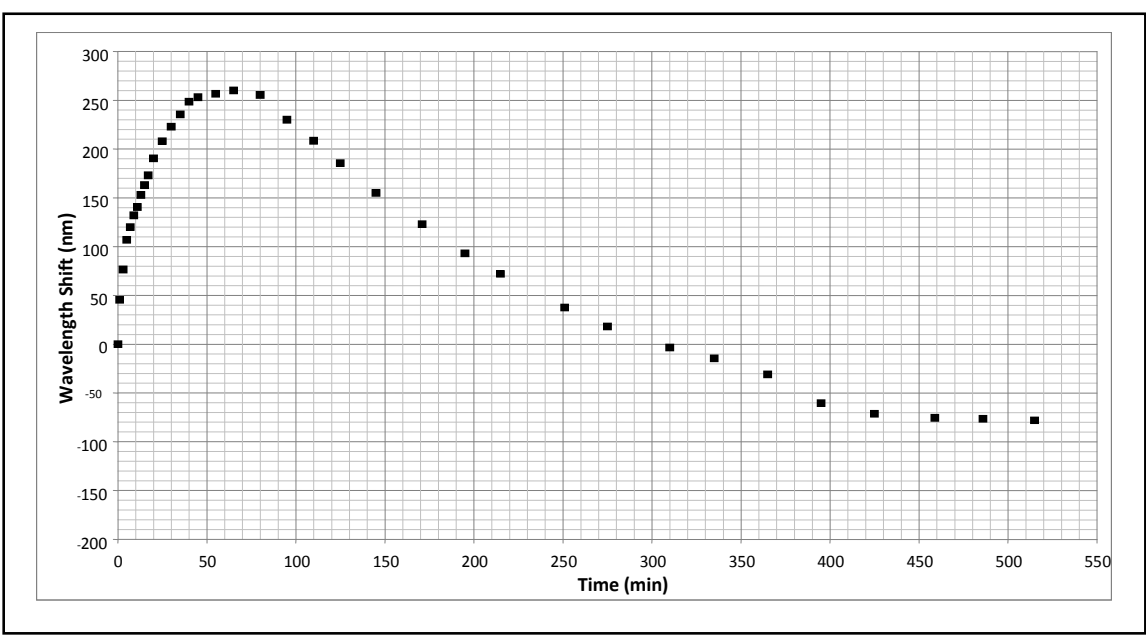

Figure 4 Shift in the resonant wavelength for coupling to one cladding mode of a single mode mPOF. 
For many applications, cross-sensitivity to moisture is of course a disadvantage. In such circumstances there are polymers available that are both suitable for fibre manufacture and photosensitive but which do not have the same affinity for water ${ }^{40}$.

\subsection{Low cost grating based sensors}

Fibre Bragg gratings do not yet represent a low cost sensing option. Various factors are contributing to the lowering of the cost of grating fabrication, such as the expiration of key early patents and the ability to write gratings directly on the drawing tower during fibre manufacture ${ }^{41}$. However the combination of single transverse mode optical sources and the high precision (better than 1 part in a million) wavelength measurement needed to obtain microstrain resolution has tended to keep costs high. When POF is used for the measurement of very high strains, it may often not be necessary to achieve microstrain resolution, and in such circumstances the use of FBGs in multimode fibre may be practical. Multimode fibre brings three advantages: grating fabrication is easier due to relaxed positional tolerances, large core fibre is compatible with broad area optical sources, e.g. edge emitting LEDs, and connection to silica fibre becomes easier. Balancing these advantages is the fact that the reflection spectrum of a FBG in multimode fibre is necessarily broadened. Figure 5 shows two gratings multiplexed in a single length of 50 micron core polymer PCF. The bandwidth of the FBGs is approximately $3 \mathrm{~nm}$ - about an order of magnitude greater than would be obtained in single mode fibre.

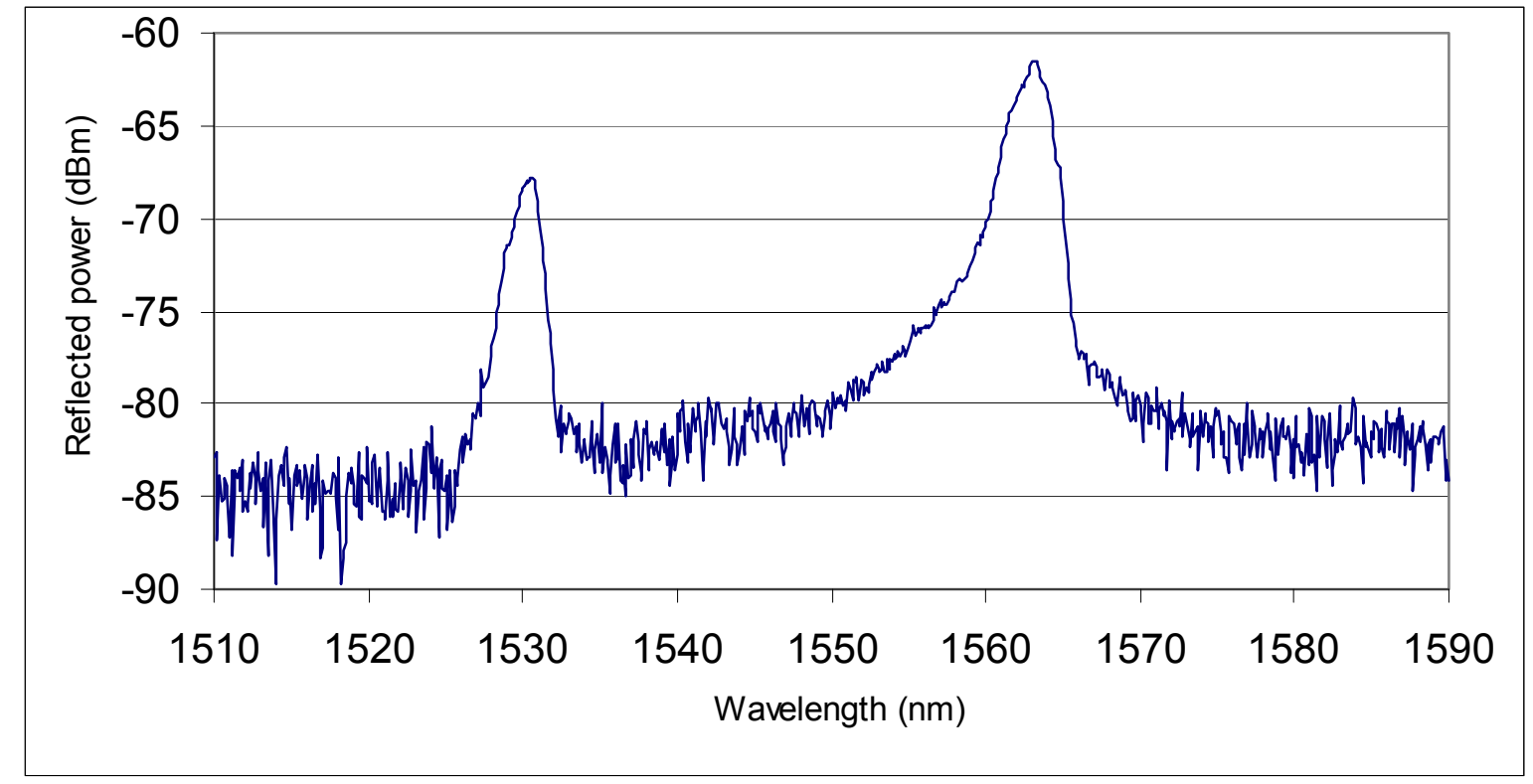

Figure 5 Reflection spectrum from two FBGs multiplexed in 50 micron core multimode photonic crystal POF (resolution bandwidth $0.1 \mathrm{~nm}$ ).

\subsection{Imaging}

An application closely related to sensing is imaging. It has been shown that either the spaces between a regular lattice of holes, or indeed the holes themselves, can guide light. This offers up the possibility of replacing expensive to fabricate fibre bundle imaging light guides with flexible mPOF. To date the most developed version of this approach is a system with 4417 pixels produced by fusing together seven, 631 hole mPOFs fabricated from PMMA after the holes had been filled by polystyrene. When pulled down to a diameter of $250 \mu \mathrm{m}$, the device provided $3 \mu \mathrm{m}$ pixels, with the resultant image being transmitted over a distance of $50 \mathrm{~cm}^{42}$.

\section{CONCLUSIONS}

The combination of polymeric fibre and the photonic crystal geometry offers the possibility to extend the range of applications for optical fibre sensors. This is a relatively new field and significant developments are anticipated, 
particularly in the area of grating based sensors and biosensors. Most research to date has focussed on fibre based on PMMA as this is most commonly used in fibres designed for telecommunications. It seems likely that research into other materials would be highly beneficial for sensing applications.

\section{ACKNOWLEDGEMENTS}

The author would like to acknowledge financial support from the UK Engineering and Physical Sciences Research Council and the European Commission (Project PHOSFOS - PHOtonic Skins For Optical Sensing)

\section{REFERENCES}

[1] A. Argyros, "Microstructured Polymer Optical Fibers," Journal of Lightwave Technology, 27(11), 1571-1579 (2009).

[2] J. C. Knight, T. A. Birks, P. S. Russell et al., "All-silica single-mode optical fiber with photonic crystal cladding," Optics Letters, 21(19), 1547-1549 (1996).

[3] T. A. Birks, J. C. Knight, and P. S. Russell, "Endlessly single-mode photonic crystal fiber," Optics Letters, 22(13), 961-963 (1997).

[4] J. C. Knight, J. Broeng, T. A. Birks et al., "Photonic band cap guidance in optical fibers," Science, 282(5393), 1476-1478 (1998).

[5] W. Daum, J. Krauser, P. E. Zamzow et al., [POF - polymer Optical Fibers for Data Communication] Springer, (2001).

[6] R. J. Bartlett, R. Philip-Chandy, P. Eldridge et al., "Plastic optical fibre sensors and devices," Transactions of the Institute of Measurement and Control, 22(5), 431-457 (2000).

[7] M. Silva-Lopez, A. Fender, W. MacPherson et al., "Strain and temperature sensitivity of a single-mode polymer optical fiber," Optics Letters, 30(23), 3129-3131 (2005).

[8] G. W. Kaye, and T. H. Laby, [Tables of physical and chemical constants] Longmann, 45 (1995).

[9] C. C. Ye, J. M. Dulieu-Barton, D. J. Webb et al., "Applications of polymer optical fibre grating sensors to condition monitoring of textiles", Proceedings of SPIE Vol. 7503, 75030M (2009)..

[10] S. Kiesel, K. Peters, T. Hassan et al., "Behaviour of intrinsic polymer optical fibre sensor for large-strain applications," Measurement Science \& Technology, 18(10), 3144-3154 (2007).

[11] D. Yang, J. Yu, X. Tao et al., "Structural and mechanical properties of polymeric optical fiber," Materials Science and Engineering, A364, 256-259 (2004).

[12] D. R. Blacket, M. Large, and A. Argyros, "High Strain sensing with mPOF and Long Period Gratings, 18th International COnference on Plastic Optical Fibre." Paper 52.

[13] N. G. Harbach, [Fiber bragg gratings in polymer optical fibers], Thesis, EPFL, Lausanne(2008).

[14] M. C. J. Large, S. Ponrathnam, A. Argyros et al., "Solution doping of microstructured polymer optical fibres," Optics Express, 12(9), 1966-1971 (2004).

[15] X. H. Yang, and L. L. Wang, "Fluorescence $\mathrm{pH}$ probe based on microstructured polymer optical fiber," Optics Express, 15(25), 16478-16483 (2007).

[16] F. M. Cox, A. Argyros, M. C. J. Large et al., "Surface enhanced Raman scattering in a hollow core microstructured optical fiber," Optics Express, 15(21), 13675-13681 (2007).

[17] A. Wang, A. Docherty, B. T. Kuhlmey et al., "Side-hole fiber sensor based on surface plasmon resonance," Optics Letters, 34(24), 3890-3892 (2009).

[18] J. B. Jensen, P. E. Hoiby, G. Emiliyanov et al., "Selective detection of antibodies in microstructured polymer optical fibers," Optics Express, 13(15), 5883-5889 (2005).

[19] G. Emiliyanov, J. B. Jensen, O. Bang et al., "Localized biosensing with Topas microstructured polymer optical fiber," Optics Letters, 32(5), 460-462 (2007).

[20] W. J. Tomlinson, I. P. Kaminow, A. Chandross et al., "Photoinduced refractive index increase in poly(methyl methacrylate) and its applications," Applied Physics Letters, 16, 486-489 (1970).

[21] I. P. Kaminow, H. P. Weber, and E. A. Chandross, "Poly(methyl methacrylate) dye laser with internal diffraction grating resonator," Applied Physics Letters, 18(11), 497 (1971).

[22] Xiong, Z., Peng et al., "Highly tunable Bragg gratings in single-mode polymer optical fibers," IEEE Photonics Technology Letters, 11(3), 352-354 (1999).

[23] H. Dobb, D. J. Webb, K. Kalli et al., "Continuous wave ultraviolet light-induced fiber Bragg gratings in fewand single-mode microstructured polymer optical fibers," Optics Letters, 30(24), 3296-3298 (2005). 
[24] Z. C. Li, H. Y. Tam, L. X. Xu et al., "Fabrication of long-period gratings in poly(methyl methacrylate-comethyl vinyl ketone-cobenzyl methacrylate)-core polymer optical fiber by use of a mercury lamp," Optics Letters, 30(10), 1117-1119 (2005).

[25] D. Sáez-Rodríguez, J. L. Cruz, I. Johnson et al., "Water diffusion into UV inscripted Long Period Grating in microstructured polymer fibre," IEEE Sensors Journal, in press (2010).

[26] D. D. Davis, T. K. Gaylord, E. N. Glytsis et al., "Long-period fibre grating fabrication with focused CO2 laser pulses," Electronics Letters, 34(3), 302-303 (1998).

[27] H. Dobb, K. Kalli, and D. J. Webb, "Temperature-insensitive long period grating sensors in photonic crystal fibre," Electronics Letters, 40(11), 657-8 (2004).

[28] S. Savin, M. J. F. Digonnet, G. S. Kino et al., "Tunable mechanically induced long-period fiber gratings," Optics Letters, 25(10), 710-712 (2000).

[29] M. Hiscocks, M. v. Eijkelenborg, A. Argyros et al., "Stable imprinting of long-period gratings in microstructured polymer optical fibre," Optics Express, 14(11), 4644-8 (2006).

[30] Z. Zhang, P. Zhao, P. Lin et al., "Thermo-optic coefficients of polymers for optical waveguide applications," Polymer, 47(14), 4893-4896 (2006).

[31] H. Y. Liu, G. D. Peng, and P. L. Chu, "Thermal tuning of polymer optical fiber Bragg gratings," IEEE Photonics Technology Letters, 13(8), 824-826 (2001).

[32] A. D. Kersey, M. A. Davis, H. J. Patrick et al., "Fiber grating sensors," Journal of Lightwave Technology, 15(8), 1442-1463 (1997).

[33] G. D. Peng, and P. L. Chu, "Polymer optical fiber photosensitivities and highly tunable fiber gratings," Fiber and Integrated Optics, 19, 277-293 (2000).

[34] D. J. Webb, and K. Kalli, "Polymer fibre Bragg gratings" in [Fiber Bragg Grating Sensors: Thirty Years from Research to Market] Bentham eBooks, in press (2010).

[35] J. Brandrup, [Polymer Handbook] Wiley, V89 (1999).

[36] K. E. Carroll, C. Zhang, D. J. Webb et al., "Thermal response of Bragg gratings in PMMA microstructured optical fibers," Optics Express, 15(14), 8844-8850 (2007).

[37] C. Jiang, M. Kuzyk, J.-L. Ding et al., "Fabrication and mechanical behavior of dye-doped polymer optical fiber," Journal of Applied Physics, 92(1), 04-Dec (2002).

[38] T. Ishigure, M. Hirai, M. Sato et al., "Graded-Index Plastic Optical Fiber with High Mechanical Properties Enabling Easy Network Installations. I,” Journal of Applied Polymer Science, 91(1), 404-409 (2003).

[39] C. Zhang, X. Chen, D. J. Webb et al., "Water detection in jet fuel using a polymer optical fibre Bragg grating", Postdeadline paper, $20^{\text {th }}$ International Conference on Optical Fibre Sensors, (2009).

[40] www.topas.com.

[41] C. G. Askins, M. A. Putnam, G. M. Williams et al., "Stepped-wavelength optical-fiber Bragg grating arrays fabricated in-line on a draw tower," Optics Letters, 19(2), 147-149 (1994).

[42] D. P. Kong, and L. L. Wang, "Ultrahigh-resolution fiber-optic image guides derived from microstructured polymer optical fiber preforms," Optics Letters, 34(16), 2435-2437 (2009). 